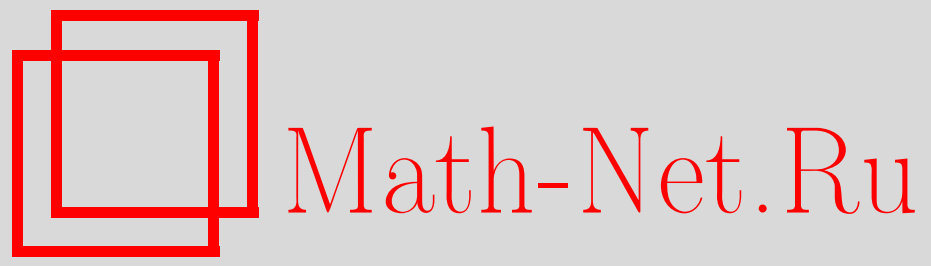

С. К. Багдасаров, Свойства $\omega$-перестановок, Функи. анализ и его прил., 1999, том 33, выпуск 3, 1-20

DOI: https://doi.org/10.4213/faa362

Использование Общероссийского математического портала MathNet.Ru подразумевает, что вы прочитали и согласны с пользовательским соглашением

http://www . mathnet.ru/rus/agreement

Параметры загрузки:

IP : 54.174 .149 .18

26 апреля 2023 г., 13:04:38

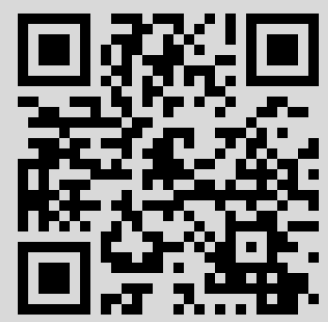


Функииональньй анализ и его приложения

1999, т. 33, вып. 3, с. 1-20

УДК 517.9

\title{
Свойства $\omega$-перестановок
}

\author{
(c) 1999. С. К. БАГДАСАРОВ
}

\section{$\S 0$. Введение}

0.1. Функциональные классы $\boldsymbol{H}^{\boldsymbol{\omega}}[\boldsymbol{a}, \boldsymbol{b}]$. Рассмотрим функциональные классы $H^{\omega}(\mathbb{I})$, впервые введенные С. М. Никольским [8].

ОПреДЕЛЕниЕ 0.1. Неубывающая функция $\omega: \mathbb{R}_{+} \rightarrow \mathbb{R}_{+}$называется выликльм модулем непрерывности, если $\omega(0)=0$ и

$$
\omega\left(\alpha t_{1}+(1-\alpha) t_{2}\right) \geqslant \alpha \omega\left(t_{1}\right)+(1-\alpha) \omega\left(t_{2}\right), \quad \forall \alpha \in(0,1), 0<t_{1}<t_{2} .
$$

ОПРЕДЕЛЕНИЕ 0.2 . Пусть $\mathbb{I}=[a, b], \tau \in \mathbb{I}$, и $\omega(\cdot)$ - выпуклый модуль непрерывности. Функииональные классьи $H^{\omega}[a, b]$ и $H_{\tau}^{\omega}(\mathbb{I})$ определяются следующим образом:

$$
\begin{aligned}
& H^{\omega}(\mathbb{I}):=\{x \in C(\mathbb{I}) \mid \omega(x ; t) \leqslant \omega(t), t \in[0,|\mathbb{I}|]\}, \\
& H_{\tau}^{\omega}(\mathbb{I}):=\left\{x \in H^{\omega}(\mathbb{I}) \mid x(\tau)=0\right\},
\end{aligned}
$$

где $\omega(x ; t)=\sup _{\left|t_{2}-t_{1}\right| \leqslant t}\left|x\left(t_{2}\right)-x\left(t_{1}\right)\right|$ — модуль непрерывности функции $x$.

В качестве примера мы упомянем выпуклые модули непрерывности $\omega_{\alpha}(t)=$ $t^{\alpha}, 0<\alpha \leqslant 1$, и гёльдеровские классы $H^{\alpha}(\mathbb{I}):=H^{\omega_{\alpha}}(\mathbb{I})$.

Пусть $\psi \in L_{1}[a, b]$ - ядро с одной переменой знака и нулевым средним на $[a, b]$ и $\Psi(x):=\int_{b}^{x} \psi(t) d t, a \leqslant x \leqslant b$. Н. П. Корнейчук [6] выразил численное решение задачи максимизации интегрального функционала $h \mapsto \int_{a}^{b} h(t) \psi(t) d t$ в классах $H^{\omega}[a, b]$ в терминах убьвающей перестановки $\Psi^{*}$ интеграла $\int_{b}^{x} \psi(t) d t$ :

$$
\sup _{h \in H^{\omega}[a, b]} \int_{a}^{b} h(t) \psi(t) d t=\int_{0}^{b-a} \Psi^{*}(t) \omega^{\prime}(t) d t .
$$

Опираясь на это свойство стандартных перестановок, в [1] автор ввел понятие $\omega$-перестановки абсолютно непрерывного ядра $\Psi$ с произвольным конечным числом точек экстремума. Напомнив основные элементы теории $\omega$-перестановок, в настоящей статье мы установим фундаментальные неравенства между стандартными убывающими перестановками, $\omega$-перестановками и $\Sigma$-перестановками Корнейчука.

0.2. Содержание статьи. В первом параграфе мы перечислим основные неравенства для убывающих перестановок. Второй параграф посвящен обсуждению уже упомянутого результата Корнейчука о максимизации интегральных функционалов в классах $H^{\omega}[a, b]$. В $\S 3$ мы опишем структуру экстремалей задачи

$$
\int_{a}^{b} h(t) \psi(t) d t \rightarrow \sup , \quad h \in H_{a}^{\omega}[a, b]
$$


и выразим значение максимума в $(\star)$ в терминах экстремальной $\omega$-nерестановкu $\Re_{\omega}(\Psi ; \cdot)$ (см. определение 3.10 ниже) ядра $\Psi(x)=\int_{b}^{x} \psi(t) d t$ следующим образом:

$$
\sup _{h \in H_{a}^{\omega}[a, b]} \int_{a}^{b} h(t) \psi(t) d t=\int_{0}^{b-a} \Re_{\omega}(\Psi ; t) \omega^{\prime}(t) d t .
$$

Наконец, в заключительном параграфе нашей работы мы установим основные отношения, связывающие три разных вида перестановок ядра $\Psi$ : стандартную убывающую перестановку $\Psi^{*}(\cdot), \Sigma$-перестановку $\Sigma(\Psi ; \cdot)$ Корнейчука (см. $\S 4)$ и $\omega$-перестановку $\Re_{\omega}(\Psi ; \cdot)$. В частности, мы опишем элементарные свойства и выведем серию неравенств для сумм, произведений и производных трех упомянутых выше видов перестановок.

\section{§1. Предварительные замечания}

В первом параграфе мы напомним читателю элементарные свойства выпуклых модулей непрерывности, перечислим интегральные неравенства, используемые в нашем анализе, и приведем хорошо известные свойства убывающих перестановок, которые будут использоваться или найдут свое обобщение в данной статье.

1.1. Выпуклые модули непрерывности. Следующие свойства выпуклых модулей непрерывности $\omega$ установлены, например, в [6].

ПРЕДЛОЖЕНИЕ 1.1. Выпукльй модуль непрерьвности $\omega-$ абсолютно непрерьвная функиия с односторонними производными

$$
\omega_{-}^{\prime}(x)=\lim _{h \rightarrow 0+} \frac{\omega(x)-\omega(x-h)}{h}, \quad \omega_{+}^{\prime}(x)=\lim _{h \rightarrow 0+} \frac{\omega(x+h)-\omega(x)}{h}, \quad x>0,
$$

удовлетворяющими неравенствам

(i) $\omega_{+}^{\prime}(x) \leqslant \omega_{-}^{\prime}(x), x>0$;

(ii) $\omega_{-}^{\prime}(x) \geqslant \omega_{-}^{\prime}(y), \omega_{+}^{\prime}(x) \geqslant \omega_{+}^{\prime}(y), 0<x<y$.

ОПРЕДЕЛЕНИЕ 1.2. Производная вылуклого модуля непрерыввности $\omega$ определяется на $\mathbb{R}_{+} \backslash\{0\}$ формулой

$$
\omega^{\prime}(u):=\frac{1}{2}\left[\omega_{+}^{\prime}(u)+\omega_{-}^{\prime}(u)\right], \quad u>0 .
$$

Следующее элементарное свойство функций из классов $H^{\omega}(\mathbb{I})$ облегчает понимание структуры экстремалей задачи $(\star)$.

ПРЕДЛОЖЕНИЕ 1.3. Если $f \in H^{\omega}[a, b]$ и для некоторых точек $\left\{a_{j}\right\}_{j=1}^{4}, a_{1}<$ $a_{2}, a_{3}<a_{4}$,

$$
\left|f\left(a_{2}\right)-f\left(a_{1}\right)\right|=\omega\left(a_{2}-a_{1}\right), \quad\left|f\left(a_{4}\right)-f\left(a_{3}\right)\right|=\omega\left(a_{4}-a_{3}\right),
$$

то или $\left(a_{1}, a_{2}\right) \cap\left[a_{3}, a_{4}\right]=\varnothing$, или $\left[a_{1}, a_{2}\right] \subseteq\left[a_{3}, a_{4}\right]$, или $\left[a_{3}, a_{4}\right] \subseteq\left[a_{1}, a_{2}\right]$.

1.2. Интегральные неравенства. Следующие неравенства используются в $\S 5$ (см. [7]). 
Лемма 1.4. Пусть $f \in L_{1}[\alpha, \beta], \int_{\alpha}^{x} f(t) d t \geqslant 0$ для всех $x \in[\alpha, \beta]$ u $g(\cdot)$ - такая неотричательная невозрастающая функция на $[\alpha, \beta]$, ито $f \cdot g \in$ $L_{1}[\alpha, \beta]$. Тогда

$$
\int_{\alpha}^{\beta} f(t) g(t) d t \geqslant 0 .
$$

ЗАмеЧАниЕ 1.5 . Если $g \in C[\alpha, \beta]$, то лемма 1.4 следует из теоремы о среднем, которая гарантирует существование такого $\tau \in(\alpha, \beta)$, что

$$
\int_{\alpha}^{\beta} f(t) g(t) d t=g(\alpha) \int_{\alpha}^{\tau} f(t) d t \geqslant 0 .
$$

ОПРеДЕлЕНИЕ 1.6. Пусть $g, h \in L_{1}[\alpha, \beta]$. Мы будем придерживаться следующих обозначений:

$$
\begin{aligned}
& g \rightarrow h \Longleftrightarrow \int_{\alpha}^{x} g(t) d t \leqslant \int_{\alpha}^{x} h(t) d t, \quad \forall x \in[\alpha, \beta], \\
& g \hookrightarrow h \Longleftrightarrow \int_{x}^{\beta} g(t) d t \leqslant \int_{x}^{\beta} h(t) d t, \quad \forall x \in[\alpha, \beta] .
\end{aligned}
$$

ЗАмЕчание 1.7. Если $\int_{\alpha}^{\beta} g(t) d t=\int_{\alpha}^{\beta} h(t) d t$, то $g \rightarrow h \Longleftrightarrow h \hookrightarrow g$.

Приведенные ниже следствия леммы 1.4 будут многократно использоваться в $\S 5$.

СЛЕДСТвИЕ 1.8. Пусть $g, h \in L_{1}[\alpha, \beta]$ таковы, ито $g \rightarrow h$. Eсли $b(\cdot)-$ неотричательная невозрастаючая функиия и $g \cdot b, h \cdot b \in L_{1}[\alpha, \beta]$, mo $g \cdot b \rightarrow h \cdot b$. В частности,

$$
\int_{\alpha}^{\beta} g(x) b(x) d x \leqslant \int_{\alpha}^{\beta} h(x) b(x) d x .
$$

СЛЕДСтвиЕ 1.9. Пусть $u, v \in L_{1}[\alpha, \beta]$ таковы, что $u \hookrightarrow v$. Если $a(\cdot)-$ неотричательная неубывающая функция и $u \cdot a, v \cdot a \in L_{1}[\alpha, \beta]$, то $u \cdot a \hookrightarrow v \cdot a$. В частности,

$$
\int_{\alpha}^{\beta} u(x) a(x) d x \leqslant \int_{\alpha}^{\beta} v(x) a(x) d x .
$$

1.3. Свойства стандартных убывающих перестановок. Соответствующие аналоги следующих свойств убывающих перестановок (см. $[4,7])$ для $\omega$-перестановок будут установлены в $§ 5$.

ПРЕДЛОЖЕНИЕ 1.10. Пусть $x$ и $y$ - измеримые функиии на $[a, b]$. Тогда

(i) $\|x\|_{L_{p}[a, b]}=\left\|x^{*}\right\|_{L_{p}[0, b-a]} \partial$ дя всех $p \geqslant 1$;

(ii) $\left(|x|^{p}\right)^{*}(t)=\left(x^{*}\right)^{p}(t), t \in[0, b-a]$;

(iii) $\int_{a}^{b} x(t) y(t) d t \leqslant \int_{0}^{b-a} x^{*}(t) y^{*}(t) d t$;

(iv) $(x+y)^{*} \rightarrow x^{*}+y^{*}$.

Отметим неравенство Харди-Литтлвуда - неравенство (iii) из этого предложения, - широко применяющееся в теории пространств, инвариантных относительно перестановок (см. [4]). 


\section{§2. Простые ядра и лемма Корнейчука}

2.1. Простые ядра и их свойства. Простое ядро можно описать как функцию $\Psi$ с носителем $[a, b]$, для которой уравнение $|\Psi(x)|=y$ имеет в точности два решения на $[a, b]$ для всех $y \in\left(0,\|\Psi\|_{C[a, b]}\right)$.

ОПРЕДЕЛЕНИЕ 2.1. Пусть $\psi \in L_{1}[a, b], \int_{a}^{b} \psi(x) d x=0$, такова, что

$$
\psi(x)<0, x \in\left[a, a^{\prime}\right], \quad \psi(x)=0, x \in\left[a^{\prime}, b^{\prime}\right], \quad \psi(x)>0, x \in\left[b^{\prime}, b\right],
$$

для некоторых $a^{\prime}, b^{\prime}, a<a^{\prime} \leqslant b^{\prime}<b$. Функция $\Psi(x)=\xi \int_{b}^{x} \psi(t) d t$ для $\xi \in$ $\{1,-1\}$ с носителем $[a, b]$ называется простым ядром (см. рис. 1$)$.

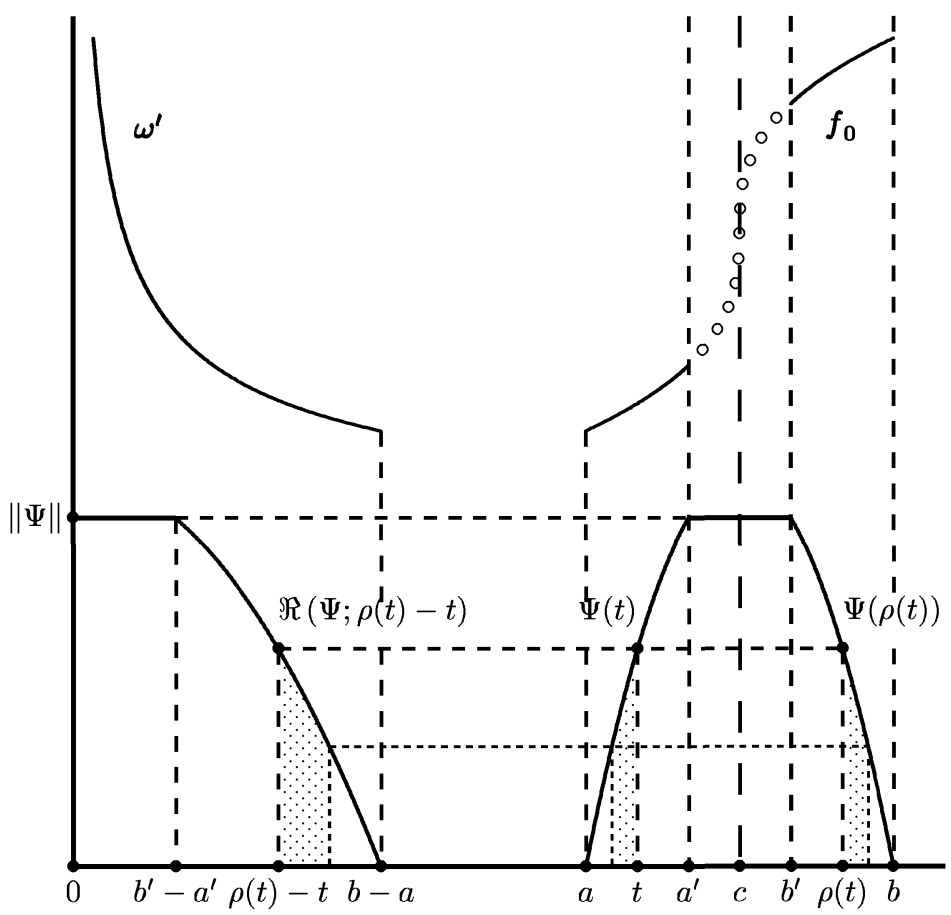

Рис. 1. Перестановка $\Re(\Psi ; \cdot)$ простого ядра $\Psi$ и функция $f_{0}$

Следующий результат (см. [6]) дает формулу для производной перестановки $\Psi^{*}$ простого ядра $\Psi$.

Лемма 2.2. Если $\Psi$ - простое ядро и $x \in\left(b^{\prime}-a^{\prime}, b-a\right)$, mo

$$
\left[\frac{d}{d x} \Psi^{*}(x)\right]^{-1}=\left[\frac{d}{d x} \Psi\left(t_{2}(x)\right)\right]^{-1}-\left[\frac{d}{d x} \Psi\left(t_{1}(x)\right)\right]^{-1}
$$

для maкux $t_{1}=t_{1}(x) \in\left(a, a^{\prime}\right) u t_{2}=t_{2}(x) \in\left(b^{\prime}, b\right)$, umo $t_{2}-t_{1}=x$.

ДокаЗАтЕльство. Пусть $h=h_{1}+h_{2}, h_{1}, h_{2}>0$, и $t_{1}-h_{1}, t_{1} \in\left[a, a^{\prime}\right]$, $t_{2}, t_{2}+h_{2} \in\left[b^{\prime}, b\right]$ таковы, что

$$
\begin{gathered}
\Psi^{*}\left(t_{2}-t_{1}\right)=\Psi\left(t_{1}\right)=\Psi\left(t_{2}\right), \\
\Psi^{*}\left(t_{2}-t_{1}+h\right)=\Psi\left(t_{1}-h_{1}\right)=\Psi\left(t_{2}+h_{2}\right) .
\end{gathered}
$$


Из (2.3) вытекает, что для $x:=t_{2}-t_{1}$ мы имеем

$$
\begin{aligned}
& h /\left(\Psi^{*}(x+h)-\Psi^{*}(x)\right) \\
& \quad=h_{1} /\left(\Psi\left(t_{1}-h_{1}\right)-\Psi\left(t_{1}\right)\right)+h_{2} /\left(\Psi\left(t_{2}+h_{2}\right)-\Psi\left(t_{2}\right)\right) .
\end{aligned}
$$

Переходя к пределу в (2.4) при $h_{1}, h_{2} \rightarrow 0$, получаем (2.2).

СЛЕДСТВИЕ 2.3. Если $\Psi$ - простое ядро, то

$$
\left|\frac{d}{d x} \Psi^{*}(x)\right| \leqslant \frac{1}{4}\left|\Psi^{\prime}\left(t_{2}(x)\right)-\Psi^{\prime}\left(t_{1}(x)\right)\right|, \quad x \in\left(b^{\prime}-a^{\prime}, b-a\right),
$$

и равенство в (2.5) имеет место тогда и только тогда, когда $\Psi^{\prime}\left(t_{1}(x)\right)=$ $-\Psi^{\prime}\left(t_{2}(x)\right)$.

2.2. Лемма Корнейчука. Лемма 2.4 Корнейчука и ее следствие (см. [6]) описывают экстремальные функции и дают значение верхней грани в (*) для производных $\psi$ простых ядер.

Лемма 2.4. Пусть $\Psi(t):=\int_{a}^{t} \psi(y) d y$ - простое ядро с производной $\psi$, удовлетворяющей условию (2.1), и $\omega$ - вьпукльй модуль непрерывности. Пусть $c:=\left(a^{\prime}+b^{\prime}\right) / 2$ и $\rho:[a, c] \rightarrow[c, b]$ определяется условиями

$$
\Psi(t)=\Psi(\rho(t)), \quad t \in\left[a, a^{\prime}\right], \quad \rho(t)=a^{\prime}+b^{\prime}-t, \quad t \in\left[a^{\prime}, c\right] .
$$

Tогда

$$
\sup _{f \in H^{\omega}[a, b]} \int_{a}^{b} f(t) \psi(t) d t=\int_{0}^{b-a} \Psi^{*}(t) \omega^{\prime}(t) d t
$$

и верхняя грань в (2.7) достигается на функииях $f_{0}(t)+C$ с производной

$$
\frac{d}{d t} f_{0}(t)= \begin{cases}\omega^{\prime}(\rho(t)-t), & a \leqslant t \leqslant c \\ \omega^{\prime}\left(t-\rho^{-1}(t)\right), & c \leqslant t \leqslant b .\end{cases}
$$

СЛЕДСТВИЕ 2.5. Функиия $f_{0}$ с производной $(d / d x) f_{0}(x)$, определенной в $(2.8)$, имеет полный модуль непрерьвности на $[0, b-a]: \omega\left(f_{0} ; t\right)=\omega(t), 0 \leqslant t \leqslant b-a$. Более того,

$$
f_{0}(\rho(t))-f_{0}(t)=\omega(\rho(t)-t), \quad 0 \leqslant t \leqslant c .
$$

В $\S 3$ мы покажем, что если $\Psi(t)=\int_{a}^{t} \psi(x) d x$ имеет конечное число точек локального экстремума (т.е. $\psi$ меняет знак конечное число раз), то $\Psi$ разлагается в конечную сумму $\Psi(t)=\sum_{i=1}^{l} \Phi_{i}(t)$ простых ядер $\Phi_{i}(\cdot)=\Phi_{i}(\omega ; \cdot)$ со свойствами

$$
\sup _{h \in H^{\omega}[a, b]} \int_{a}^{b} h(t) \psi(t) d t=\sum_{i=1}^{l} \sup _{h \in H^{\omega}[a, b]} \int_{a}^{b} h(t) \Phi_{i}^{\prime}(t) d t .
$$

\section{§3. Экстремумы интегральных функционалов и перестановки $\Re_{\omega}(\Psi ; \cdot)$}

В 33 мы обсудим структуру экстремальных функций задачи ( $)$ и введем понятие $\omega$-перестановок $\Re_{\omega}(\Psi ; \cdot)$ абсолютно непрерывных ядер с конечным числом локальных экстремумов. Все результаты данного параграфа установлены автором в $[1,2]$. 


\section{1. Обозначения и определения.}

ОБознАчениЕ 3.1. Обозначение $I=\oslash$ используется для интервалов $I=$ $[\gamma, \gamma]$ с пустой внутренностью.

ОПРедЕлЕниЕ 3.2. Пусть $m \in\{-1,0,+1\}$ и $n \in \mathbb{N}$. Тогда $\psi \in L_{1}[a, b]$ nринадлежит $\mathscr{M}_{n}^{m}[a, b]$ при $n \geqslant 2$ в том и только том случае, когда $\operatorname{sign} \int_{a}^{b} \psi(x) d x=m$ и

$$
\operatorname{meas}\left\{t \in\left[\alpha_{i-1}, \alpha_{i}\right] \mid(-1)^{i} \psi(t)>0\right\}=\alpha_{i}-\alpha_{i-1}, \quad i=1, \ldots, n,
$$

для некоторых $a=\alpha_{0}<\alpha_{1}<\cdots<\alpha_{n-1}<\alpha_{n}=b$.

ОПРЕДЕЛЕНИЕ 3.3. Для $n \in \mathbb{N}$ множества индексов $\left\{J_{i}(n)\right\}_{i \leqslant n},\left\{L_{i}\right\}_{i \leqslant n}$ и пар индексов $\mathscr{P}(n)$ определяются следующим образом:

$$
\begin{aligned}
J_{i}(n) & =\left\{\begin{array}{l}
\varnothing \quad \text { для } i=n-2, n-1, n, \text { если } n \geqslant 3, \text { и для всех } i \leqslant n, \text { если } n<3, \\
\{j=i+1+2 k, k \in \mathbb{N} \mid j \leqslant n\}, \quad 1 \leqslant i \leqslant n-3,
\end{array}\right. \\
L_{i} & =\left\{\begin{array}{l}
\varnothing, \quad i=1,2,3, \\
\{l=i-1-2 k, k \in \mathbb{N} \mid l \geqslant 1\}, \quad 4 \leqslant i \leqslant n,
\end{array}\right. \\
\mathscr{P}(n) & =\left\{(i, j) \in \mathbb{N}^{2} \mid 1 \leqslant i \leqslant n-3, j \in J_{i}(n)\right\} .
\end{aligned}
$$

Например, $J_{1}(8)=\{4,6,8\}, J_{2}(8)=\{5,7\}, J_{3}(8)=\{6,8\}, J_{4}(8)=\{7\}, J_{5}(8)=$ $\{8\}$ и $L_{4}=\{1\}, L_{5}=\{2\}, L_{6}=\{1,3\}, L_{7}=\{2,4\}, L_{8}=\{1,3,5\}$.

3.2. $\boldsymbol{V}_{\boldsymbol{n}}^{\boldsymbol{m}}$-разбиения ограниченных интервалов. Строение экстремалей задачи $(\star)$ характеризуется в терминах специальных разбиений интервала $[a, b]$.

ОПредЕлЕниЕ 3.4. Пусть $n \in \mathbb{N}, m \in\{-1,0,+1\}$. Разбиение $\mathscr{V}=\left\{A_{i}, B_{i}\right.$, $\left.C_{i}, D_{i}\right\}_{i=1}^{n}$ промежутка $[a, b]$ на подынтервалы $\left\{B_{i j}, C_{j i}\right\}_{(i, j) \in \mathscr{P}(n)}$ называется $V_{n}^{m}$-разбиением, если выполняются следующие условия:

(A) $C_{i}=\left[\gamma_{4 i-4}, \gamma_{4 i-3}\right], D_{i}=\left[\gamma_{4 i-3}, \gamma_{4 i-2}\right], B_{i}=\left[\gamma_{4 i-2}, \gamma_{4 i-1}\right], A_{i}=\left[\gamma_{4 i-1}, \gamma_{4 i}\right]$, $i=1, \ldots, n$, для таких $\left\{\gamma_{i}\right\}_{i=0}^{4 n}$, что $a=\gamma_{0} \leqslant \gamma_{1} \leqslant \ldots \leqslant \gamma_{4 n}=b$;

(B) $A_{n}=\odot, C_{i}=\odot, i=1,2,3, B_{i}=\odot, i=n-2, n-1, n$;

$\left(\mathrm{C}_{1}\right) m=0 \Longrightarrow D_{i}=\boxminus, i=1, \ldots, n$;

$\left(\mathrm{C}_{2}\right) m=-1 \Longrightarrow D_{2 k}=\odot, k=1, \ldots,[n / 2]$;

$\left(\mathrm{C}_{3}\right) m=+1 \Longrightarrow D_{2 k-1}=\boxminus, k=1, \ldots,\lceil n / 2\rceil$;

(D) $B_{i}=\bigcup_{j \in J_{i}(n)} B_{i j}, 1 \leqslant i \leqslant n-3$, где $B_{i j}=\left[\xi_{i}\left(\frac{j-i+1}{2}\right), \xi_{i}\left(\frac{j-i-1}{2}\right)\right], j \in J_{i}(n)$, для таких $\left\{\xi_{i}(k)\right\}_{k=1}^{\left|J_{i}(n)\right|}$, что $\gamma_{4 i-2}=\xi_{i}\left(\left|J_{i}(n)\right|\right) \leqslant \ldots \leqslant \xi_{i}(2) \leqslant \xi_{i}(1)=$ $\gamma_{4 i-1}$

(Е) $C_{i}=\bigcup_{l \in L_{i}} C_{i l}, 4 \leqslant i \leqslant n$, где $C_{i l}=\left[\varkappa_{i}\left(\frac{i-l-1}{2}\right), \varkappa_{i}\left(\frac{i-l+1}{2}\right)\right], l \in L_{i}$, для таких $\left\{\varkappa_{i}(k)\right\}_{k=1}^{\left|L_{i}\right|}$, что $\gamma_{4 i-4}=\varkappa_{i}(1) \leqslant \varkappa_{i}(2) \leqslant \ldots \leqslant \varkappa_{i}\left(\left|L_{i}\right|\right)=\gamma_{4 i-3}$.

ПримеР 3.5. Перечислим все атомы $\left\{A_{i}, B_{i}, C_{i}, D_{i}\right\}_{i=1}^{n} V_{n}^{m}$-разбиений интервала $[a, b]$ за исключением вырожденных промежутков $A_{n},\left\{B_{i}\right\}_{i=n-2}^{n},\left\{C_{i}\right\}_{i=1}^{3}$ : $n=2: D_{1} A_{1} D_{2}$

$n=3: D_{1} A_{1} D_{2} A_{2} D_{3}$;

$n=4: D_{1} B_{1} A_{1} D_{2} A_{2} D_{3} A_{3} C_{4} D_{4}$

$n=5: D_{1} B_{1} A_{1} D_{2} B_{2} A_{2} D_{3} A_{3} C_{4} D_{4} A_{4} C_{5} D_{5}$; 
$n=6: D_{1} B_{1} A_{1} D_{2} B_{2} A_{2} D_{3} B_{3} A_{3} C_{4} D_{4} A_{4} C_{5} D_{5} A_{5} C_{6} D_{6}$;

$n \geqslant 7: D_{1} B_{1} A_{1} D_{2} B_{2} A_{2} D_{3} B_{3} A_{3} C_{k} D_{k} B_{k} A_{k} C_{n-2} D_{n-2} A_{n-2} C_{n-1} D_{n-1} A_{n-1} C_{n} D_{n}$, где $k$ меняется от 4 до $n-3$.

Рис. 2 проясняет порядок атомов в $V_{8}^{0}$-разбиениях.

$\begin{array}{llllllllllll}B_{16} & A_{1} & B_{25} & B_{38} & A_{3} & B_{47} & C_{52} & A_{5} & C_{61} & C_{74} & A_{7} & C_{83}\end{array}$ $\begin{array}{lllllllllllll}B_{18} & B_{14} & B_{27} & A_{2} & B_{36} & C_{41} & A_{4} & B_{58} & C_{63} & A_{6} & C_{72} & C_{85} & C_{81}\end{array}$

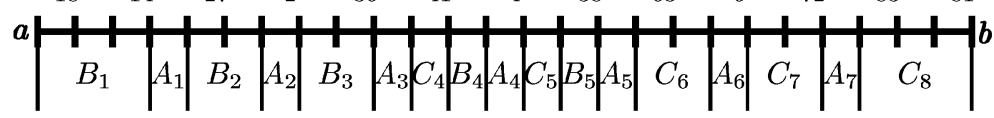

Рис. 2. $V_{8}^{0}$-разбиение

3.3. Теорема о максимизации интегральных функционалов. Через $\mathscr{X}(E ; t)$ обозначим характеристическую функцию множества $E \subset \mathbb{R}$,

$$
\mathscr{X}(E ; t)= \begin{cases}1, & t \in E, \\ 0, & t \notin E .\end{cases}
$$

Teорема 3.6. Пусть $\psi \in \mathscr{M}_{n}^{m}[a, b], m \in\{-1,0,+1\}, u\left\{\alpha_{i}\right\}_{i=0}^{n}-$ mочки neремены знака лдра $\psi$ в смысле определения 3.2. Тогда существует экстремаль $x_{\omega, \psi}$ в задаче $(\star)$ и $V_{n}^{m}$-разбиение $\mathscr{V}$ интервала $[a, b]$ со следующими свойствамu:

(A) $\alpha_{i} \in A_{i}, i=1, \ldots, n-1$;

(B) $\int_{B_{i j} \cup C_{j i}} \psi(t) d t=0,(i, j) \in \mathscr{P}(n)$;

(C) $\int_{A_{i}} \psi(t) d t=0, i=1, \ldots, n-1$;

(D $\left.\mathrm{D}_{1}\right) m=-1 \Longrightarrow x_{\omega, \psi}(t)=-\omega(t-a), t \in D_{2 k-1} \neq \odot, k=1, \ldots,\lceil n / 2\rceil$;

$\left(\mathrm{D}_{2}\right) m=1 \Longrightarrow x_{\omega, \psi}(t)=\omega(t-a), t \in D_{2 k} \neq \odot, k=1, \ldots,[n / 2]$;

(E) для всех $(i, j) \in \mathscr{P}(n)$ функция $x_{\omega, \psi}$ әкстремальна в задаче

$$
\begin{gathered}
\int_{a}^{b} h(t) \psi_{i j}(t) d t \rightarrow \sup , \quad h \in H^{\omega}[a, b], \\
\psi_{i j}(t):=\psi(t) \cdot \mathscr{X}\left(B_{i j} \cup C_{j i} ; t\right), \quad t \in[a, b] ;
\end{gathered}
$$

(F) для всех $i=1, \ldots, n-1$ функция $x_{\omega, \psi}$ әкстремальна в задаче

$$
\begin{array}{cc}
\int_{a}^{b} h(t) \psi_{i}(t) d t \rightarrow \sup , & h \in H^{\omega}[a, b], \\
\psi_{i}(t):=\psi(t) \cdot \mathscr{X}\left(A_{i} ; t\right), \quad & t \in[a, b] .
\end{array}
$$

3.4. Элементарные свойства экстремалей задачи $(\star)$. Опираясь на формулы леммы Корнейчука, в этом разделе мы введем понятие $\omega$-перестановок $\Re_{\omega}(\Psi ; \cdot)$.

ПрЕДЛОЖЕНИЕ 3.7. Для $(i, j) \in \mathscr{P}(n) u k=1, \ldots, n-1$

$$
\Psi_{i j}(t)=\int_{b}^{t} \psi_{i j}(y) d y, \quad \Psi_{k}(t)=\int_{b}^{t} \psi_{k}(y) d y
$$

являются простыми ядрами в смысле определения 2.1 . 
ДоказАтельство. Действительно, из включений $\alpha_{i} \in A_{i}=:\left[\alpha_{i}^{1}, \alpha_{i}^{2}\right], i=$ $1, \ldots, n-1$, и порядка атомов в $V_{n}^{m}$-разбиении (см. пример 3.5) мы заключаем, что

$$
\operatorname{sign} \psi(t)= \begin{cases}(-1)^{i} & \text { на } \begin{cases}B_{i j}, & (i, j) \in \mathscr{P}(n), \\ {\left[\alpha_{i}^{1}, \alpha_{i}\right],} & i=1, \ldots, n-1,\end{cases} \\ (-1)^{i+1} & \text { на } \begin{cases}C_{j i}, & (i, j) \in \mathscr{P}(n), \\ {\left[\alpha_{i}, \alpha_{i}^{2}\right],} & i=1, \ldots, n-1,\end{cases} \end{cases}
$$

где $\left[\alpha_{i}^{1}, \alpha_{i}^{2}\right]:=A_{i}, i=1, \ldots, n-1$, и

$$
\operatorname{sign} \psi(t)=\left\{\begin{aligned}
-1 & \text { на } D_{2 i-1}, i=1, \ldots,\lceil n / 2\rceil, \\
1 & \text { на } D_{2 i}, i=1, \ldots,[n / 2] .
\end{aligned}\right.
$$

Таким образом, из (3.8) и утверждений (B) и (C) теоремы 3.6 следует, что $\left\{\Psi_{i j}\right\}_{(i, j) \in \mathscr{P}(n)}$ и $\left\{\Psi_{i}\right\}_{i=1}^{n-1}$ - простые ядра.

Лемма Корнейчука дает следующие формулы для производной $d x_{\omega, \psi}(t) / d t$ :

$$
\frac{d}{d t} x_{\omega, \psi}(t)= \begin{cases}(-1)^{i+1} \omega^{\prime}\left(\rho_{i j}(t)-t\right), & t \in B_{i j}, \\ (-1)^{i+1} \omega^{\prime}\left(t-\rho_{i j}^{-1}(t)\right), & t \in C_{j i},\end{cases}
$$

для всех $(i, j) \in \mathscr{P}(n)$, где $\rho_{i j}: B_{i j} \rightarrow C_{j i}$ определена из уравнения

$$
\Psi_{i j}(t)=\Psi_{i j}\left(\rho_{i j}(t)\right), \quad t \in B_{i j}, \rho_{i j}(t) \in C_{j i},
$$

и

$$
\frac{d}{d t} x_{\omega, \psi}(t)= \begin{cases}(-1)^{i+1} \omega^{\prime}\left(\rho_{i}(t)-t\right), & t \in\left[\alpha_{i}^{1}, \alpha_{i}\right], \\ (-1)^{i+1} \omega^{\prime}\left(t-\rho_{i}^{-1}(t)\right), & t \in\left[\alpha_{i}, \alpha_{i}^{2}\right],\end{cases}
$$

где $\rho_{i}:\left[\alpha_{i}^{1}, \alpha_{i}\right] \rightarrow\left[\alpha_{i}, \alpha_{i}^{2}\right]$ определена из уравнения

$$
\Psi_{i}(t)=\Psi_{i}\left(\rho_{i}(t)\right), \quad t \in\left[\alpha_{i}^{1}, \alpha_{i}\right], \rho_{i}(t) \in\left[\alpha_{i}, \alpha_{i}^{2}\right] .
$$

Схематические графики функций $x_{\omega, \psi}, \psi \in \mathscr{M}_{5}^{+1}[a, b]$, и $\Psi$ даны на рис. 3. Формулы (3.10), (3.12) приводят нас к следующей теореме единственности.

ПреДЛОЖЕНИЕ 3.8. Если $\psi \in \mathscr{M}_{n}^{m}[a, b]$, то задача (*) имеет единственное решение.

Из свойства $(2.9)$ функций $\left\{\rho_{i j}\right\}_{(i j) \in \mathscr{P}(n)}$ из уравнения $(3.11)$ и $\left\{\rho_{i}\right\}_{i=1}^{n-1}$ из уравнения (3.13) вытекает, что

$$
\begin{aligned}
x_{\omega, \psi}\left(\rho_{i j}(t)\right)-x_{\omega, \psi}(t) & =\omega\left(\rho_{i j}(t)-t\right), & & t \in B_{i j},(i, j) \in \mathscr{P}(n), \\
x_{\omega, \psi}\left(\rho_{i}(t)\right)-x_{\omega, \psi}(t) & =\omega\left(\rho_{i}(t)-t\right), & & t \in A_{i}, i=1, \ldots, n-1 .
\end{aligned}
$$

Сочетание формул (3.14) с предложением 1.3 приводит нас к следующему результату.

ПреДЛОЖЕНИЕ 3.9. Пусть $B_{i_{1} j_{1}} \neq \bullet u B_{i_{2} j_{2}} \neq \odot$. Тогда или $\left(i_{1}, j_{1}\right) \cap\left[i_{2}, j_{2}\right]=$ $\varnothing$, или $\left[i_{1}, j_{1}\right] \subseteq\left[i_{2}, j_{2}\right]$, или $\left[i_{2}, j_{2}\right] \subseteq\left[i_{1}, j_{1}\right]$. 


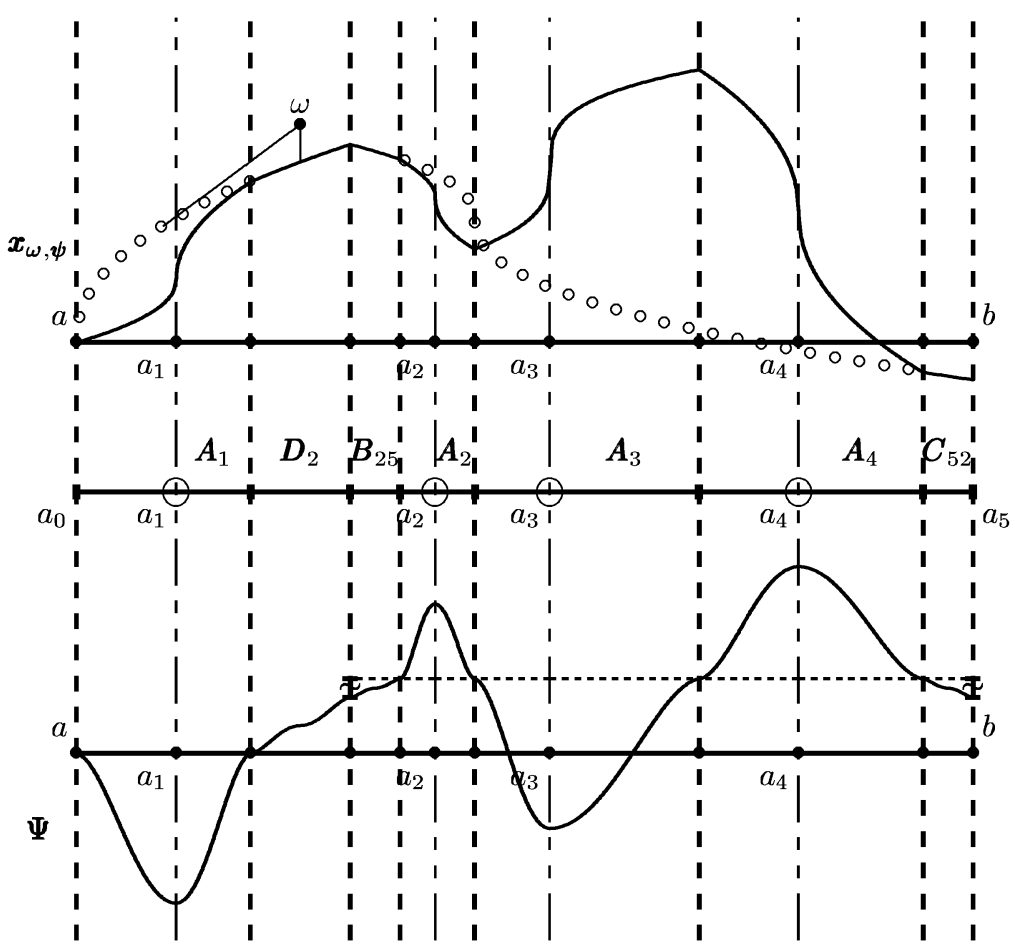

Рис. 3. $V_{5}^{+1}$-разбиение и графики функций $x_{\omega, \psi}$ и $\Psi$ для $\psi \in \mathscr{M}_{5}^{+1}[a, b]$

ОПРЕДЕЛЕНИЕ 3.10. Перестановка, или $\omega$-перестановка, $\Re_{\omega}(\Psi ; \cdot)$ определяется следующим образом:

$$
\begin{aligned}
\Re_{\omega}(\Psi ; x)= & \sum_{i=1}^{n-1} \Psi_{i}^{*}(x)+\sum_{(i, j) \in \mathscr{P}(n)} \Psi_{i, j}^{*}(x) \\
& \quad+|\Psi(x+a)| \sum_{i=0}^{n} \mathscr{X}\left(D_{i} ; x+a\right), \quad 0 \leqslant x \leqslant b-a,
\end{aligned}
$$

где простые ядра $\left\{\Psi_{i}\right\}_{i=1}^{n-1}$ и $\left\{\Psi_{i, j}\right\}_{(i, j) \in \mathscr{P}(n)}$ введены формулами (3.7).

Формула (2.8), примененная к простым ядрам из (3.7), дает численное решение задачи $(\star)$.

СлЕДСТВИЕ 3.11. Если $\omega-$ выпуклый модуль непрерывности, то

$$
\sup _{h \in H_{a}^{\omega}[a, b]} \int_{a}^{b} h(t) \psi(t) d t=\int_{0}^{b-a} \Re_{\omega}(\Psi ; x) \omega^{\prime}(x) d x .
$$

В [1] (см. также [2]) мы установили следующий результат.

ПредЛОЖЕНИЕ 3.12. Пусть $\psi \in \mathscr{M}_{n}^{0}[a, b], n \geqslant 3, x_{\omega, \psi}$ - экстремаль задачи

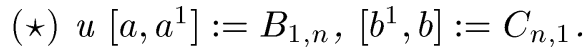

1. Существует такая точка $c \in\left(a^{1}, b^{1}\right)$, ито $\Psi\left(a^{1}\right)=\Psi(c)=\Psi\left(b^{1}\right)$ u

$$
c:=\max \left\{\tau \in\left(a^{1}, b^{1}\right) \mid x_{\omega, \psi}(\tau)-x_{\omega, \psi}\left(a^{1}\right)=\omega\left(\tau-a^{1}\right)\right\} .
$$


2. Пусть

$$
\begin{gathered}
\psi_{1}(t):=\psi(t) \mathscr{X}\left(B_{1, n} \cup C_{n, 1} ; t\right), \quad \psi_{2}(t):=\psi(t) \mathscr{X}\left(\left[a^{1}, c\right] ; t\right), \\
\psi_{3}(t)=\psi(t) \mathscr{X}\left(\left[c, b^{1}\right] ; t\right),
\end{gathered}
$$

$u$

Тогда

$$
\Psi_{k}(x)=\int_{a}^{x} \psi_{k}(t) d t, \quad x \in[a, b], k=1,2,3 .
$$

$$
\begin{gathered}
\sup _{h \in H^{\omega}[a, b]} \int_{a}^{b} h(t) \psi(t) d t=\sum_{i=1}^{3} \sup _{h \in H^{\omega}[a, b]} \int_{a}^{b} h(t) \Psi_{i}^{\prime}(t) d t \\
\Re_{\omega}(\Psi ; x)=\sum_{i=1}^{3} \Re_{\omega}\left(\Psi_{i} ; x\right), \quad x \in[0, b-a] .
\end{gathered}
$$

\section{§4. Вспомогательные конструкции}

В $\S \S 4,5$ мы зафиксируем ядро $\psi \in \mathscr{M}_{n}^{0}[a, b], n \in \mathbb{N}$. Как и раньше, положим $\Psi(x):=\int_{a}^{x} \psi(t) d t, a \leqslant x \leqslant b$. С этими ядрами свяжем точки $\left\{\alpha_{i}\right\}_{i=1}^{n-1}$ перемены знака функции $\psi$ и нули $\left\{b_{i}\right\}_{i=0}^{r}$ функции $\Psi$, упорядоченные по возрастанию: $a=: \alpha_{0}<\alpha_{1}<\cdots<\alpha_{n-1}<\alpha_{n}:=b, a=b_{0}<b_{1}<\cdots<b_{r}=: b$.

4.1. $\Sigma$-перестановки Корнейчука. $\Sigma$-разбиение Корнейчука представляет собой естественный метод разложения ядра $\Psi$ в сумму простых ядер [6].

ОПРЕДЕЛЕНИЕ 4.1. Пусть $\rho(\Psi):=\left(\Psi\left(\alpha_{0}\right), \Psi\left(\alpha_{1}\right), \ldots, \Psi\left(\alpha_{n-1}\right)\right)$ — вектор локальных экстремумов функции $\Psi$ и $\pi(\Psi)=\left(\pi_{0}, \ldots, \pi_{n-1}\right) \in \mathbb{R}^{n}$ - его возрастающая перестановка $\left(\pi_{0}=\min _{a \leqslant t \leqslant b} \Psi(t), \pi_{n-1}=\max _{a \leqslant t \leqslant b} \Psi(t)\right)$. Для $t \in[a, b]$ и $k=1, \ldots, n-1$ положим (см. рис. 4 )

$$
\Psi_{k}(t)= \begin{cases}\min \left\{\max \left\{\Psi(t), \pi_{k-1}\right\}, \pi_{k}\right\}-\pi_{k-1}, & \pi_{k}>0, \\ \min \left\{\max \left\{\Psi(t), \pi_{k-1}\right\}, \pi_{k}\right\}-\pi_{k}, & \pi_{k} \leqslant 0 .\end{cases}
$$

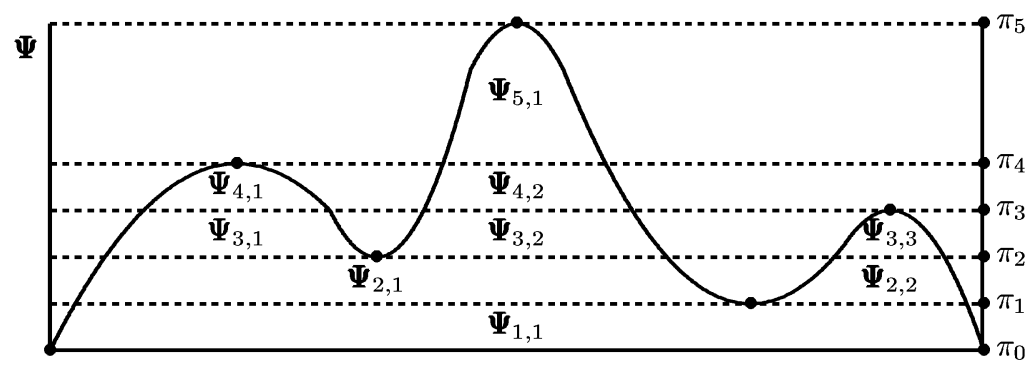

Рис. 4. इ-разложение Корнейчука

Тогда $\Psi(\cdot)=\sum_{k=1}^{n-1} \Psi_{k}(\cdot)$ и каждая из функций $\Psi_{k}(t)$ является суммой простых ядер $\left\{\Psi_{k, i}(\cdot)\right\}_{i=1}^{l_{k}}$ с неперекрывающимися носителями. $\Sigma$-разбиение $u$ 
$\Sigma$-перестановка $\Sigma(\Psi ; \cdot)$ ядра $\Psi$ определяются следующим образом:

$$
\Psi(t)=\sum_{k=1}^{n-1} \sum_{i=1}^{l_{k}} \Psi_{k, i}(t), \quad t \in[a, b], \quad \Sigma(\Psi ; t)=\sum_{k=1}^{n-1} \sum_{i=1}^{l_{k}} \Psi_{k, i}^{*}(t), \quad t \in \mathbb{R}_{+} .
$$

Мы упомянем некоторые очевидные свойства $\Sigma$-перестановок:

(i) $\|\Sigma(\Psi ; \cdot)\|_{L_{1}[0, b-a]}=\|\Psi\|_{L_{1}[a, b]}$;

(ii) $\Sigma(\Psi ; 0)=\frac{1}{2} \bigvee_{a}^{b} \Psi=\frac{1}{2}\|\psi\|_{L_{1}[a, b]}$;

(iii) $\Sigma\left(|\Psi|^{p} ; x\right) \leqslant(\Sigma(\Psi ; x))^{p}, x \in[0, b-a], p \geqslant 1$;

(iv) если $\Psi(c)=0, c \in(a, b)$, то $\Sigma(\Psi ; \cdot)=\Sigma\left(\left.\Psi\right|_{[a, c]} ; \cdot\right)+\Sigma\left(\left.\Psi\right|_{[c, b]} ; \cdot\right)$.

ПреДЛОжЕНИЕ 4.2. Равенство в (4.3)(iii) имеет место для всех $x \in[0, b-a]$ тогда и только тогда, когда $p=1$ или $\Psi$ имеет $n$ нулей $\left\{b_{i}\right\}_{i=1}^{r}$, чередуюшихся с точками $\left\{\alpha_{i}\right\}_{i=1}^{n-1}$ перемень знаков функиии $\Psi^{\prime}$ :

$$
b_{i} \leqslant \alpha_{i+1} \leqslant b_{i+1}, \quad i=0, \ldots, n-1 .
$$

Из определения вытекает, что перестановки $\Re_{\omega}(\Psi ; \cdot)$ и $\Sigma(\Psi ; \cdot)$ ядра $\Psi \in$ $\mathscr{M}_{n}^{0}[a, b]$ совпадают со стандартной неубывающей перестановкой $\Psi^{*}$ тогда и только тогда, когда $n=2$, т.е. $\Psi$ - простое ядро. В следующем разделе мы обсудим условия для тождества $\Sigma(\Psi ; \cdot) \equiv \Re_{\omega}(\Psi ; \cdot)$.

\section{2. Критерий тривиальности $V_{n}^{0}$-разбиений.}

ОПрЕДЕЛЕНИЕ 4.3. Экстремальное $V_{n}^{0}$-разбиение $\mathscr{V}$ задачи $(\star)$ для $\psi \in$ $\mathscr{M}_{n}^{0}[a, b]$ называется тривиальным разбиением, если

$$
B_{i j}=\boxminus, \quad C_{j i}=\bullet, \quad \forall(i, j) \in \mathscr{P}(n) .
$$

Тривиальность разбиения $\mathscr{V}$ эквивалентна следующему представлению ее атомов:

$$
A_{i}=\left[b_{i-1}, b_{i}\right], \quad i=1, \ldots, n-1, \quad B_{i}=C_{i}=\left\{b_{i-1}\right\}, \quad i=1, \ldots, n .
$$

ПРЕДЛОЖЕНИЕ 4.4. Пусть $\psi \in \mathscr{M}_{n}^{0}[a, b] u\left\{b_{i}\right\}_{i=0}^{n-1}-$ нули ядра $\Psi$, удовлетворяющие условию (4.4). Экстремальное разбиение $\mathscr{V}=\left\{\left\{A_{i}, B_{i}, C_{i}\right\}_{i=1}^{n}\right\}$ тривиально тогда и только тогда, когда

$$
\sum_{l=i}^{j-1}(-1)^{i+l} \omega\left(b_{l+1}-b_{l}\right) \leqslant \omega\left(b_{j}-b_{i}\right), \quad 1 \leqslant i<j \leqslant n, j-i=2 k+1, k \in \mathbb{N} .
$$

ДокАЗАТЕЛЬСтво. Предположим, что $\mathscr{V}$ - тривиальное разбиение, т. е. выполнены условия (4.6). Из экстремальности функций $x_{\omega, \psi}$ в задачах (3.5)-(3.6) и свойства (2.9) вытекает, что

$$
x_{\omega, \psi}\left(b_{i}\right)-x_{\omega, \psi}\left(b_{i-1}\right)=(-1)^{i-1} \omega\left(b_{i}-b_{i-1}\right), \quad i=1, \ldots, n-1,
$$

и, следовательно,

$$
x_{\omega, \psi}\left(b_{j}\right)-x_{\omega, \psi}\left(b_{i}\right)=\sum_{l=i}^{j-1}(-1)^{l} \omega\left(b_{l+1}-b_{l}\right), \quad 0 \leqslant i<j \leqslant n-1 .
$$


Ввиду (4.9) условия (4.7) необходимы для включения $x_{\omega, \psi} \in H^{\omega}[a, b]$. Для доказательства достаточности предположим, напротив, что выполнены условия (4.7) и сушествуют такие точки $v \in\left[b_{i-1}, b_{i}\right]$ и $w \in\left[b_{j-1}, b_{j}\right], i<j$, что

$$
\left|x_{\omega, \psi}(w)-x_{\omega, \psi}(v)\right|>\omega(w-v) .
$$

Введем индексы $I \in\{0,1\}, L \in\{0,1\}$ и $l, m$ посредством формул

$$
\begin{gathered}
(-1)^{I}=\operatorname{sign}\left((-1)^{i-1}\left(x_{\omega, \psi}(w)-x_{\omega, \psi}(v)\right)\right), \quad L=[i-j] \quad(\bmod 2), \\
l=i-(I-1), \quad m=j+(L-I), \quad i, j \in\{0,1\} .
\end{gathered}
$$

Заметим, что $m \in J_{l}(n):=\{k=l+2 r+1, r \in \mathbb{N} \mid k \leqslant n\}$. Покажем, что наше допущение (4.10) приводит к неравенствам

$$
(-1)^{l}\left(x_{\omega, \psi}\left(b_{m}\right)-x_{\omega, \psi}\left(b_{l}\right)\right)>\omega\left(b_{m}-b_{l}\right),
$$

противоречащим (4.7). Рассмотрим, например, случай $I=0, L=0$. Введем функции

$$
\begin{array}{ll}
f_{1}(t)=(-1)^{i-1}\left(x_{\omega, \psi}\left(b_{i}\right)-\omega\left(b_{i}-t\right)\right), & t \in\left[b_{i-1}, b_{i}\right], \\
f_{2}(t)=(-1)^{i-1}\left(x_{\omega, \psi}\left(b_{j-1}\right)+\omega\left(t-b_{j-1}\right)\right), & t \in\left[b_{j-1}, b_{j}\right] .
\end{array}
$$

Из включения $x_{\omega, \psi} \in H^{\omega}[a, b]$ и равенств $f_{1}\left(b_{i}\right)=x_{\omega, \psi}\left(b_{i}\right), f_{2}\left(b_{j-1}\right)=$ $x_{\omega, \psi}\left(b_{j-1}\right)$ следует, что

$$
\begin{aligned}
(-1)^{i}\left(f_{1}(t)-x_{\omega, \psi}(t)\right) \geqslant 0, & t \in\left[b_{i-1}, b_{i}\right], \\
(-1)^{j-1}\left(f_{2}(t)-x_{\omega, \psi}(t)\right) \geqslant 0, & t \in\left[b_{j-1}, b_{j}\right] .
\end{aligned}
$$

Значит, существуют точки $y \in\left[v, b_{i}\right], z \in\left[b_{j-1}, b_{j}\right]$, такие, что

$$
f_{1}(y)=x_{\omega, \psi}(v) \quad \text { и } \quad f_{2}(z)=x_{\omega, \psi}(w) .
$$

Из определения (4.16) и неравенства (4.10) вытекает, что

$$
\begin{aligned}
(-1)^{i-1}\left(f_{2}(z)-f_{2}(y)\right) & =(-1)^{i-1}\left(x_{\omega, \psi}(w)-x_{\omega, \psi}(v)\right) \\
& >\omega(w-v) \geqslant \omega(z-y) .
\end{aligned}
$$

Согласно (4.14),

$$
f_{1}\left(b_{i-1}\right)=x_{\omega, \psi}\left(b_{i-1}\right) \quad \text { и } \quad f_{2}\left(b_{j}\right)=x_{\omega, \psi}\left(b_{j}\right) .
$$

Последовательно используя (4.14), (4.17) и выпуклость функции $\omega$, мы приходим к цепочке неравенств

$$
\begin{aligned}
(-1)^{i-1} & {\left[x_{\omega, \psi}\left(b_{j}\right)-x_{\omega, \psi}\left(b_{i-1}\right)\right]=(-1)^{i-1}\left[f_{1}\left(b_{j}\right)-f_{2}\left(b_{i-1}\right)\right] } \\
& =(-1)^{i-1}\left(\left[f_{2}\left(b_{j}\right)-f_{2}(z)\right]+\left[f_{2}(z)-f_{1}(y)\right]+\left[f_{1}(y)-f_{1}\left(b_{i-1}\right)\right]\right) \\
& >\left[\omega\left(b_{j}-b_{j-1}\right)-\omega\left(z-b_{j-1}\right)\right]+\omega(z-y)+\left[\omega\left(b_{i}-b_{i-1}\right)-\omega\left(b_{i}-y\right)\right] \\
& \geqslant\left[\omega\left(b_{j}-y\right)-\omega(z-y)\right]+\omega(z-y)+\omega\left(b_{i}-b_{i-1}\right)-\omega\left(b_{i}-y\right) \\
& =\left[\omega\left(b_{j}-y\right)-\omega\left(b_{i}-y\right)\right]+\omega\left(b_{i}-b_{i-1}\right) \\
& \geqslant\left[\omega\left(b_{j}-b_{i-1}\right)-\omega\left(b_{i}-b_{i-1}\right)\right]+\omega\left(b_{i}-b_{i-1}\right)=\omega\left(b_{j}-b_{i-1}\right),
\end{aligned}
$$

противоречащей включению $x_{\omega, \psi} \in H^{\omega}[a, b]$.

Неравенство (4.13), противоречащее (4.7), в остальных трех случаях выводится аналогичным образом. 
4.3. Производные перестановок $\boldsymbol{\Sigma}(\boldsymbol{\Psi} ; \cdot)$ и $\Re_{\boldsymbol{\omega}}(\boldsymbol{\Psi} ; \cdot)$. Пусть $\left\{\widehat{\Psi}_{i}\right\}_{i=1}^{k}$ и $\left\{\widetilde{\Psi}_{i}\right\}_{i=1}^{m}$ - такие наборы простых ядер, что

$$
\Psi=\sum_{i=1}^{k} \widehat{\Psi}_{i}=\sum_{i=1}^{m} \widetilde{\Psi}_{i}, \quad \Sigma(\Psi ; \cdot)=\sum_{i=1}^{k} \widehat{\Psi}_{i}^{*}(\cdot), \quad \Re_{\omega}(\Psi ; \cdot)=\sum_{i=1}^{m} \widetilde{\Psi}_{i}^{*}(\cdot) .
$$

Для $i=1, \ldots, k$ положим $\left[\hat{\alpha}_{i}, \hat{\beta}_{i}\right]:=\operatorname{supp} \widehat{\Psi}_{i}$, и пусть $\left[\hat{\alpha}_{i}^{\prime}, \hat{\beta}_{i}^{\prime}\right]$ 一 интервал в $\left[\hat{\alpha}_{i}, \hat{\beta}_{i}\right]$, где $\widehat{\Psi}_{i}^{\prime}$ обращается в нуль. Аналогично, для $l=1, \ldots, m$ положим $\left[\tilde{\alpha}_{l}, \tilde{\beta}_{l}\right]:=$ $\operatorname{supp} \widetilde{\Psi}_{l}$, и пусть $\left[\tilde{\alpha}_{l}^{\prime}, \tilde{\beta}_{l}^{\prime}\right]$ 一 интервал в $\left[\tilde{\alpha}_{l}, \tilde{\beta}_{l}\right]$, где $\widetilde{\Psi}_{l}^{\prime}$ обращается в нуль. Пусть $\hat{\rho}_{i}:\left[\hat{\alpha}_{i}, \hat{\alpha}_{i}^{\prime}\right] \mapsto\left[\hat{\beta}_{i}^{\prime}, \hat{\beta}_{i}\right]$ и $\tilde{\rho}_{l}:\left[\tilde{\alpha}_{l}, \tilde{\alpha}_{l}^{\prime}\right] \mapsto\left[\tilde{\beta}_{l}^{\prime}, \tilde{\beta}_{l}\right]$ - отображения, определенные уравнениями

$$
\widehat{\Psi}_{i}(t)=\widehat{\Psi}_{i}\left(\hat{\rho}_{i}(t)\right), \quad i=1, \ldots, k, \quad \widetilde{\Psi}_{l}(t)=\widetilde{\Psi}_{l}\left(\tilde{\rho}_{l}(t)\right), \quad l=1, \ldots, m .
$$

Для всех $x \in[a, b]$ мы определим множества пар

$$
\begin{aligned}
& \widehat{\Lambda}(x):=\left\{\left(t_{i}, \hat{\rho}_{i}\left(t_{i}\right)\right) \mid t_{i} \in\left[\hat{\alpha}_{i}, \hat{\alpha}_{i}^{\prime}\right]: \hat{\rho}_{i}\left(t_{i}\right)-t_{i}=x, i=1, \ldots, k\right\}, \\
& \widetilde{\Lambda}(x):=\left\{\left(t_{l}, \tilde{\rho}_{l}\left(t_{l}\right)\right) \mid t_{l} \in\left[\tilde{\alpha}_{l}, \tilde{\alpha}_{l}^{\prime}\right]: \tilde{\rho}_{l}\left(t_{l}\right)-t_{l}=x, l=1, \ldots, k\right\},
\end{aligned}
$$

Оба множества $\widehat{\Lambda}(x)$ и $\widetilde{\Lambda}(x)$ обладают тем свойством, что для любых двух пар $(a, b),(c, d)$ из этих множеств интервалы $[a, b],[c, d]$ длины $x$ не имеют общих внутренних точек. Действительно, это свойство для $\widetilde{\Lambda}(x)$ вытекает из одного из трех соотношений

$$
\left(\hat{\alpha}_{i}, \hat{\beta}_{i}\right) \cap\left[\hat{\alpha}_{j}, \hat{\beta}_{j}\right]=\varnothing, \quad\left[\hat{\alpha}_{i}, \hat{\beta}_{i}\right] \subset\left[\hat{\alpha}_{j}^{\prime}, \hat{\beta}_{j}^{\prime}\right], \quad\left[\hat{\alpha}_{j}, \hat{\beta}_{j}\right] \subset\left[\hat{\alpha}_{i}^{\prime}, \hat{\beta}_{i}^{\prime}\right], \quad 1 \leqslant i<j \leqslant k,
$$

непосредственно следующих из предложения 3.9. С другой стороны, любые два интервала в $\widehat{\Lambda}(x)$ разделены одной из точек $\alpha_{j}, j=1, \ldots, n-1$. Ввиду леммы 2.2 производные перестановок $\Sigma(\Psi ; \cdot)$ и $\Re_{\omega}(\Psi ; \cdot)$ даются формулами

$$
\begin{aligned}
\frac{d}{d x} \Sigma(\Psi ; x) & =-\sum_{(a, b) \in \widehat{\Lambda}(x)}\left[\left|\Psi^{\prime}(a)\right|^{-1}+\left|\Psi^{\prime}(b)\right|^{-1}\right]^{-1}, \\
\frac{d}{d x} \Re_{\omega}(\Psi ; x) & =-\sum_{(c, d) \in \widetilde{\Lambda}(x)}\left[\left|\Psi^{\prime}(c)\right|^{-1}+\left|\Psi^{\prime}(d)\right|^{-1}\right]^{-1} .
\end{aligned}
$$

Лемма 4.5. Пусть $F=\left\{\left[a_{i}, b_{i}\right]\right\}_{i=1}^{k}$ - семейство таких неперекрьваюшихся интервалов в $[a, b]$, что $b_{i}-a_{i} \leqslant c, i=1, \ldots, k, u$ любые два промежутка семейства $F$ разделень нулем функиии $\Psi$. Тогда

$$
\sum_{i=1}^{k} \int_{a_{i}}^{b_{i}}|\Psi(x)| d x \leqslant \int_{0}^{c} \Sigma(\Psi ; x) d x .
$$

Доказательство (см. также [6]) может быть получено индукцией по числу нулей функции $\Psi$ и основано на применении свойства $(4.3)(\mathrm{iv})$.

4.4. $\Lambda$-ядра. При заданных $\Psi \in \mathscr{M}_{2 n}^{0}[a, b], n \in \mathbb{N}$, и $\lambda \in\left[\max \left\{\Psi\left(\alpha_{1}\right)\right.\right.$, $\left.\left.\Psi\left(\alpha_{2 n-1}\right)\right\}, 0\right]$ прямая $y=\lambda$ пересекает график $y=\Psi(x)$ в точках $\alpha_{\lambda}$ на $\left(0, \alpha_{1}\right)$ 
и $\beta_{\lambda}$ на $\left(\alpha_{2 n-1}, b\right)$. Более точно,

$$
\alpha_{\lambda}=\min \{\xi \in[a, b] \mid \Psi(\xi)=\lambda\}, \quad \beta_{\lambda}=\max \{\xi \in[a, b] \mid \Psi(\xi)=\lambda\} .
$$

Введем множества

$$
\begin{gathered}
E_{\lambda}^{1}:=\{x \in[a, b] \mid \Psi(x) \geqslant 0\}, \quad E_{\lambda}^{3}:=\{x \in[a, b] \mid \lambda \leqslant \Psi(x)<0\}, \\
E_{\lambda}^{2}:=\{x \in[a, b] \mid \Psi(x)<\lambda\},
\end{gathered}
$$

и ядра

$$
\begin{gathered}
\Phi_{\lambda}^{1}(x):=\Psi(x) \mathscr{X}\left(E_{\lambda}^{1} ; x\right), \quad \Phi_{\lambda}^{3}(x):=(\Psi(x)-\lambda) \mathscr{X}\left(E_{\lambda}^{3} ; x\right), \\
\Phi_{\lambda}^{2}(x):=\Psi(x) \mathscr{X}\left(E_{\lambda}^{2} ; x\right)+\lambda \mathscr{X}\left(E_{\lambda}^{3} ; x\right), \quad x \in[a, b] .
\end{gathered}
$$

ОПРЕДЕЛЕНИЕ 4.6. Введем $\lambda-я \partial p a$

$$
\begin{array}{ll}
\Psi_{\lambda}^{1}(x):=(\Psi(x)-\lambda) \mathscr{X}\left(\left[\alpha_{\lambda}, \beta_{\lambda}\right] ; x\right), & x \in[a, b], \\
\Psi_{\lambda}^{2}(x):=\Psi(x) \mathscr{X}\left(\left[a, \alpha_{\lambda}\right] \cup\left[\beta_{\lambda}, b\right] ; x\right)+\lambda \mathscr{X}\left(\left(\alpha_{\lambda}, \beta_{\lambda}\right) ; x\right), & x \in[a, b] .
\end{array}
$$

По определению $\Psi=\Phi_{\lambda}^{1}+\Psi_{\lambda}^{2}$.

Лемма 4.7. Для всех $\lambda \in\left[\max \left\{\Psi\left(\alpha_{1}\right), \Psi\left(\alpha_{2 n-1}\right)\right\}, 0\right]$

$$
\int_{x}^{b-a} \Sigma(\Psi ; t) d t \leqslant \int_{x}^{b-a} \sum_{k=1}^{2} \Sigma\left(\Psi_{\lambda}^{k} ; t\right) d t, \quad x \in[0, b-a] .
$$

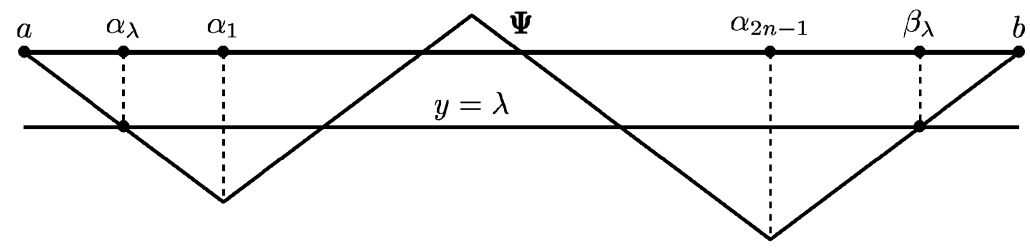

Рис. 5. Иллюстрация к лемме 4.7

ДоказАТЕльство. По определению (рис. 5 проясняет ситуацию) для $x \in$ $[0, b-a]$

$$
\Sigma(\Psi ; x)=\sum_{k=1}^{3} \Sigma\left(\Phi_{\lambda}^{k} ; x\right), \quad \Sigma\left(\Psi_{\lambda}^{1} ; x\right) \geqslant \Sigma\left(\Phi_{\lambda}^{1} ; x\right)+\Sigma\left(\Phi_{\lambda}^{3} ; x\right) .
$$

Из (4.28) вытекает, что

$$
\sum_{k=1}^{2} \Sigma\left(\Psi_{\lambda}^{k} ; x\right)-\Sigma(\Psi ; x) \geqslant \Sigma\left(\Psi_{\lambda}^{2} ; x\right)-\Sigma\left(\Phi_{\lambda}^{2} ; x\right) .
$$

Однако $0 \geqslant \Phi_{\lambda}^{2}(x) \geqslant \Psi_{\lambda}^{2}(x), x \in[a, b]$, что в сочетании со свойством (iii) из предложения 5.3 ниже означает, что

$$
\int_{x}^{b-a} \Sigma\left(\Phi_{\lambda}^{2} ; t\right) d t \leqslant \int_{x}^{b-a}\left(\Phi_{\lambda}^{2}\right)^{*}(t) d t \leqslant \int_{x}^{b-a}\left(\Psi_{\lambda}^{2}\right)^{*}(t) d t=\int_{x}^{b-a} \Sigma\left(\Psi_{\lambda}^{2} ; t\right) d t .
$$


Комбинируя (4.30) и (4.29), приходим к (4.27).

СлЕДСТВИЕ 4.8. Если $\omega-$ выпукльй модуль непрерьвности, то $\Sigma(\Psi ; \cdot) \hookrightarrow$ $\Re_{\omega}(\Psi ; \cdot)$.

ДокАЗАТЕльСтво. Пусть $\Psi^{\prime} \in \mathscr{M}_{n}^{0}[a, b]$. Применим индукцию по $n$. Если $n=2$, т.е. $\Psi$ - простое ядро, то $\Re_{\omega}(\Psi ; \cdot) \equiv \Sigma(\Psi ; \cdot) \equiv \Psi^{*}(\cdot)$. Предположив, что результат верен для всех ядер $\Psi$ с $\Psi^{\prime} \in \mathscr{M}_{k}^{0}[\alpha, \beta]$ при $k \leqslant n$, мы установим отношение $\Sigma(\Psi ; \cdot) \hookrightarrow \Re_{\omega}(\Psi ; \cdot)$ для всех ядер $\Psi$ с $\Psi^{\prime} \in \mathscr{M}_{n+1}^{0}[a, b]$.

В наших рассуждениях мы опираемся на результаты (3.17), (3.19), (3.20) предложения 3.12. Пусть $\lambda^{*}:=\Psi\left(a^{1}\right)$. Тогда $\lambda^{*}$-ядра (4.26) выражаются через $\Psi_{i}, i=1,2,3$, из (3.18):

$$
\Psi_{\lambda^{*}}^{1}(x)=\Psi_{2}(x)+\Psi_{3}(x), \quad \Psi_{\lambda^{*}}^{2}(x)=\Psi_{1}(x), \quad x \in[a, b] .
$$

Ядра $\Psi_{2} \in \mathscr{M}_{l}^{0}\left[a^{1}, c\right], \Psi_{3} \in \mathscr{M}_{n+2-l}^{0}\left[a^{1}, c\right]$ имеют неперекрываюшиеся носители, а $\Psi_{1}$ - простое ядро. Следовательно, опираясь на лемму 4.7, предложение 3.12 , разложение из (4.31) и наши предположения индукции, мы приходим к цепочке неравенств

$$
\begin{aligned}
\int_{x}^{b-a} \Sigma(\Psi ; t) d t & \leqslant \int_{x}^{b-a} \sum_{k=1}^{2} \Sigma\left(\Psi_{\lambda^{*}}^{k} ; t\right) d t=\int_{x}^{b-a} \sum_{k=1}^{3} \Sigma\left(\Psi_{k} ; t\right) d t \\
& \leqslant \int_{x}^{b-a} \sum_{k=1}^{3} \Re_{\omega}\left(\Psi_{k} ; t\right) d t=\int_{x}^{b-a} \Re_{\omega}(\Psi ; t) d t
\end{aligned}
$$

завершающей доказательство.

\section{$\S 5$. Неравенства для перестановок}

В главной части статьи мы выведем разнообразные неравенства, управляющие поведением $\Sigma$-, $\omega$ - и стандартных перестановок, и раскроем тесную связь между всеми тремя видами перестановок.

В этом параграфе аббревиатура в.м.н. обозначает выпуклый модуль непрерьвности.

Первый результат немедленно вытекает из леммы Корнейчука.

ЛЕмма 5.1. Пусть $\left\{\Phi_{i}\right\}_{i=1}^{l}-$ набор простьх ядер на $[a, b] u$

$$
\Psi(t):=\sum_{i=1}^{l} \Phi_{i}(t), \quad t \in[a, b], \quad \Phi(t)=\sum_{i=1}^{l} \Phi_{i}^{*}(t), \quad t \in[0, b-a] .
$$

Тогда

$$
\sup _{h \in H^{\omega}[a, b]} \int_{a}^{b} h(t) \Psi^{\prime}(t) d t \leqslant \int_{0}^{b-a} \Phi(t) \omega^{\prime}(t) d t .
$$

Определения $3.10,4.1$ перестановок $\Re_{\omega}(\Psi ; \cdot)$ и $\Sigma(\Psi ; \cdot)$ в сочетании с леммой 5.1 приводят нас к следуюшим заключениям.

СЛЕДСТвИЕ 5.2. Пусть $\omega, \mu-$ любые два в.м.н. $и \sigma, \pi-$ любъе два таких в.м.н., ито $\sigma(x) \leqslant \pi(x), x \in[0, b-a]$. Тогда

(i) $\int_{0}^{b-a} \Re_{\omega}(\Psi ; t) \omega^{\prime}(t) d t \leqslant \int_{0}^{b-a} \Re_{\mu}(\Psi ; t) \omega^{\prime}(t) d t$, 
(ii) $\int_{0}^{b-a} \Re_{\omega}(\Psi ; t) \omega^{\prime}(t) d t \leqslant \int_{0}^{b-a} \Sigma(\Psi ; t) \omega^{\prime}(t) d t$,
(iii) $\int_{0}^{b-a} \Re_{\pi}(\Psi ; t) \sigma^{\prime}(t) d t \leqslant \int_{0}^{b-a} \Re_{\sigma}(\Psi ; t) \pi^{\prime}(t) d t$.

ДокАЗАТЕЛЬСтво. Поскольку каждая из функций $\Sigma(\Psi ; \cdot)$ и $\Re_{\omega}(\Psi ; \cdot)$ является суммой убывающих перестановок простых ядер, первые два неравенства - прямое следствие леммы 5.1. Установим неравенство (iii). Интегрируя по частям и опираясь на равенства $\Re_{\omega}(\Psi ; b-a)=0, \omega(0)=0$ и неравенство (ii), мы получаем

$$
\begin{aligned}
\int_{0}^{b-a} \Re_{\pi}(\Psi ; t) \sigma^{\prime}(t) d t & =-\int_{0}^{b-a} \frac{d}{d t} \Re_{\pi}(\Psi ; t) \sigma(t) d t \leqslant-\int_{0}^{b-a} \frac{d}{d t} \Re_{\pi}(\Psi ; t) \pi(t) d t \\
& =\int_{0}^{b-a} \Re_{\pi}(\Psi ; t) \pi^{\prime}(t) d t \leqslant \int_{0}^{b-a} \Re_{\sigma}(\Psi ; t) \pi^{\prime}(t) d t .
\end{aligned}
$$

Вспомним определение 1.6 “---” - и “ฯ”-отношений для суммируемых функций.

ПРЕДЛОЖЕНИЕ 5.3. Пусть $\omega-$ в.м.н. Тогда

(i) $\Psi^{*} \rightarrow \Sigma(\Psi ; \cdot)$;

(ii) $\Psi^{*} \rightarrow \Re_{\omega}(\Psi ; \cdot)$;

(iii) $\Sigma(\Psi ; \cdot) \hookrightarrow \Psi^{*}$.

ДоказАТЕльство. Например, выведем (ii). Если $\Psi(\cdot)=\sum_{i=1}^{l} \Phi_{i}(\cdot)-$ разложение ядра $\Psi$ в сумму простых ядер $\left\{\Phi_{i}\right\}_{i=1}^{l}$, такое, что $\Re_{\omega}(\Psi ; \cdot)=$ $\sum_{i=1}^{l} \Phi_{i}^{*}(\cdot)$, то, согласно (iv) из предложения 1.10 , мы имеем

$$
\int_{0}^{x} \Psi^{*}(t) d t=\int_{0}^{x}\left(\sum_{i=1}^{l} \Phi_{i}\right)^{*}(t) d t \leqslant \int_{0}^{x} \sum_{i=1}^{l} \Phi_{i}^{*}(t) d t=\int_{0}^{x} \Re_{\omega}(\Psi ; t) d t .
$$

Вывод (i) аналогичен доказательству (ii), а неравенства (iii) следуют из (i), поскольку $\int_{0}^{b-a} \Psi^{*}(x) d x=\int_{0}^{b-a} \Sigma(\Psi ; x) d x=\|\Psi\|_{L_{1}[a, b]}$.

Заметим, что отношение $\Sigma(\Psi ; \cdot) \hookrightarrow \Re_{\omega}(\Psi ; \cdot)$ было установлено ранее в следствии 4.8 .

Следующее неравенство между интегралами произведений функций и их $\omega$ - и $\Sigma$-перестановок естественным образом обобщает неравенство Харди-Литтлвуда (см. неравенство (iii) предложения 1.10).

ПРеДЛОЖЕНИЕ 5.4. Если $\Psi_{1} \in \mathscr{M}_{l}^{0}[a, b], \Psi_{2} \in \mathscr{M}_{k}^{0}[a, b] u \omega, \sigma-$ любъе два в.м.н., mo

(i) $\int_{a}^{b} \Psi_{1}(x) \Psi_{2}(x) d x \leqslant \int_{0}^{b-a} \Re_{\omega}\left(\Psi_{1} ; t\right) \Re_{\sigma}\left(\Psi_{2} ; t\right) d t$,

(ii) $\int_{a}^{b} \Psi_{1}(x) \Psi_{2}(x) d x \leqslant \int_{0}^{b-a} \Sigma\left(\Psi_{1} ; t\right) \Re_{\sigma}\left(\Psi_{2} ; t\right) d t$,

(iii) $\int_{a}^{b} \Psi_{1}(x) \Psi_{2}(x) d x \leqslant \int_{0}^{b-a} \Sigma\left(\Psi_{1} ; t\right) \Sigma\left(\Psi_{2} ; t\right) d t$. 
ДоказАТЕльство. Заметим, что стандартные перестановки $\Psi^{*}$ и $\omega$-перестановки $\Re_{\omega}(\Psi ; \cdot)$ являются невозрастающими функциями. Последовательно используя неравенство (iii) из предложения 1.10 , отношения $\Psi_{1}^{*}-\rightarrow \Re_{\omega}\left(\Psi_{1} ; \cdot\right)$ и $\Psi_{2}^{*} \rightarrow \Re_{\sigma}\left(\Psi_{2} ; \cdot\right)$ предложения 5.3 и опираясь на предложение 1.8 , мы выводим цепочку неравенств

$$
\begin{aligned}
\int_{a}^{b} \Psi_{1}(t) \Psi_{2}(t) d t & \leqslant \int_{0}^{b-a} \Psi_{1}^{*}(t) \Psi_{2}^{*}(t) d t \\
& \leqslant \int_{0}^{b-a} \Re_{\omega}\left(\Psi_{1} ; t\right) \Psi_{2}^{*}(t) d t \leqslant \int_{0}^{b-a} \Re_{\omega}\left(\Psi_{1} ; t\right) \Re_{\sigma}\left(\Psi_{2} ; t\right) d t,
\end{aligned}
$$

приводящую к доказываемому результату. Другие два неравенства проверяются аналогичным образом.

Следующий результат представляет собой прямое следствие предложений $1.8,1.9$ и 5.3.

СлЕДСТВИЕ 5.5. Пусть $\omega-$ в.м.н.

1. Если $g(\cdot)$ - неотричательная и невозрастающая функиия на $[0, b-a]$, то $\Psi^{*}(\cdot) g(\cdot) \rightarrow \Sigma(\Psi ; \cdot) g(\cdot)$ u $\Psi^{*}(\cdot) g(\cdot) \rightarrow \Re_{\omega}(\Psi ; \cdot) g(\cdot)$. В частности,

$$
\begin{aligned}
& \int_{0}^{b-a} \Psi^{*}(t) g(t) d t \leqslant \int_{0}^{b-a} \Sigma(\Psi ; t) g(t) d t \\
& \int_{0}^{b-a} \Psi^{*}(t) g(t) d t \leqslant \int_{0}^{b-a} \Re_{\omega}(\Psi ; t) g(t) d t .
\end{aligned}
$$

2. Если $c(\cdot)$ - неотричательнал и неубьвающая функиия на $[0, b-a]$, то $\Sigma(\Psi ; \cdot) c(\cdot) \hookrightarrow \Psi^{*}(\cdot) c(\cdot)$ и $\Sigma(\Psi ; \cdot) c(\cdot) \hookrightarrow \Re_{\omega}(\Psi ; \cdot) c(\cdot)$. В частности,

$$
\begin{aligned}
& \int_{0}^{b-a} \Sigma(\Psi ; t) c(t) d t \leqslant \int_{0}^{b-a} \Re_{\omega}(\Psi ; t) c(t) d t, \\
& \int_{0}^{b-a} \Sigma(\Psi ; t) c(t) d t \leqslant \int_{0}^{b-a} \Psi^{*}(t) c(t) d t .
\end{aligned}
$$

ЗАМЕЧАнИЕ 5.6. В частности, неравенства (5.3) справедливы для производной $\omega^{\prime}$ любого выпуклого модуля непрерывности в качестве функции $g$.

Обратимся теперь к неравенствам между $L_{p}$-нормами перестановок. В соответствии с равенством (i) предложения $1.10\left\|\Psi^{*}\right\|_{L_{p}[0, b-a]}=\|\Psi\|_{L_{p}[a, b]}$ для всех $p \geqslant 1$. Следующие результаты показывают, что $L_{p}$-нормы перестановок $\Sigma(\psi ; \cdot)$ и $\Re_{\omega}(\Psi ; \cdot)$ мажорируют $\|\Psi\|_{L_{p}[a, b]}$.

СлЕДСТВИЕ 5.7. Пусть $1 \leqslant p \leqslant \infty u \omega-$ в.м.н. Тогда

$$
\|\Psi\|_{L_{p}[a, b]} \leqslant\|\Sigma(\Psi ; \cdot)\|_{L_{p}[0, b-a]}, \quad\|\Psi\|_{L_{p}[a, b]} \leqslant\left\|\Re_{\omega}(\Psi ; \cdot)\right\|_{L_{p}[0, b-a]} \cdot
$$

ДокАЗАТЕльСтво. Положив $g(\cdot)=\left(\Psi^{*}(\cdot)\right)^{p-1}$ в $(5.3)$ и опираясь на следствие 5.5 в сочетании с неравенством Гёльдера, мы приходим к оценкам

$$
\begin{aligned}
\int_{0}^{b-a} \Psi^{*}(t)\left(\Psi^{*}\right)^{p-1}(t) d t & \leqslant \int_{0}^{b-a} \Sigma(\Psi ; t)\left(\Psi^{*}\right)^{p-1}(t) d t \\
& \leqslant\left(\int_{0}^{b-a}\left(\Psi^{*}\right)^{p}(t) d t\right)^{1-1 / p}\left(\int_{0}^{b-a} \Sigma^{p}(\Psi ; t) d t\right)^{1 / p}
\end{aligned}
$$


И

$$
\begin{aligned}
\int_{0}^{b-a} \Psi^{*}(t)\left(\Psi^{*}\right)^{p-1}(t) d t & \leqslant \int_{0}^{b-a} \Re_{\omega}(\Psi ; t)\left(\Psi^{*}\right)^{p-1}(t) d t \\
& \leqslant\left(\int_{0}^{b-a}\left(\Psi^{*}\right)^{p}(t) d t\right)^{1-1 / p}\left(\int_{0}^{b-a} \Re_{\omega}^{p}(\Psi ; t) d t\right)^{1 / p}
\end{aligned}
$$

эквивалентным неравенствам (5.8).

ЗАмечАниЕ 5.8 . Что касается связи между $L_{p}$-нормами $\Sigma$ - и $\omega$-перестановок, можно лишь утверждать, что $\|\Sigma(\Psi ; \cdot)\|_{L_{1}[a, b]} \leqslant\left\|\Re_{\omega}(\Psi ; \cdot)\right\|_{L_{1}[a, b]}$ и

$$
\left\|\Re_{\omega}(\Psi ; \cdot)\right\|_{C[0, b-a]}=\|\Sigma(\Psi ; \cdot)\|_{C[0, b-a]}=\frac{1}{2}\left\|\Psi^{\prime}\right\|_{L_{1}[a, b]} \cdot
$$

Более того, можно найти ядро $\Psi$, в.м.н. $\omega$ и $p>1$, такие, что

$$
\|\Sigma(\Psi ; \cdot)\|_{L_{p}[0, b-a]}>\left\|\Re_{\omega}(\Psi ; \cdot)\right\|_{L_{p}[0, b-a]} \cdot
$$

Пусть $\Psi$ - четное ядро на $[-10,10]$, заданное при $t \in[0,10]$ формулой $\Psi(t)=$ $\min \{t, 10-t\}$, и пусть $\omega(t)=\sqrt{t}$. Тогда $\Sigma(\Psi ; t)=(10-t)_{+}$и

$$
\begin{aligned}
\Re_{\omega}(t)=( & \left.10-\frac{3}{2} t\right) \mathscr{X}([0,2] ; t)+(9-t) \mathscr{X}([2,8] ; t) \\
& +\mathscr{X}([8,18] ; t)+\left(10-\frac{1}{2} t\right) \mathscr{X}([18,20] ; t) .
\end{aligned}
$$

Прямые вычисления показывают, что $\|\Sigma(\Psi ; \cdot)\|_{L_{p}[0,20]} \asymp 10^{1+1 / p} /(p+1)$, в то время как $\left\|\Re_{\omega}(\Psi ; \cdot)\right\|_{L_{p}[0,20]} \asymp(2 / 3) 10^{1+1 / p} /(p+1)$, когда $p \rightarrow \infty$.

ОПРЕДЕлЕнИЕ 5.9. Функции $f, g \in C[c, d]$ состоят в отношении $f \diamond g$, если или $f \equiv g$ на $[c, d]$, или существуют такие точки $x, y \in[c, d]$, что $f(x)>g(x)$ и $f(y)<g(y)$.

ЗАмЕЧАНИЕ 5.10. Другими словами, $f \diamond g$ тогда и только тогда, когда или $f \equiv g$, или существуют две неотрицательные функции $h_{1}, h_{2} \in L_{1}[c, d]$, такие, чTо

$$
\int_{c}^{d} f(x) h_{1}(x) d x>\int_{c}^{d} g(x) h_{1}(x) d x, \quad \int_{c}^{d} f(x) h_{2}(x) d x<\int_{c}^{d} g(x) h_{2}(x) d x .
$$

СлЕДСТВИЕ 5.11. Если $\omega$ и

(i) $\Re_{\omega}(\Psi ; \cdot) \diamond \Re_{\mu}(\Psi ; \cdot)$;

(ii) $\Re_{\omega}(\Psi ; \cdot) \diamond \Sigma(\Psi ; \cdot)$;

(iii) $\Sigma(\Psi ; \cdot) \diamond \Psi^{*}(\cdot)$;

(iv) $\int_{0}^{x} \Re_{\omega}(\Psi ; t) d t \diamond \int_{0}^{x} \Sigma(\Psi ; t) d t$.

ДокАЗАТЕЛЬСТво. Ввиду неравенства (i) следствия 5.2 мы имеем

$$
\begin{aligned}
& \int_{0}^{b-a} \Re_{\omega}(\Psi ; t) \omega^{\prime}(t) d t \leqslant \int_{0}^{b-a} \Re_{\mu}(\Psi ; t) \omega^{\prime}(t) d t, \\
& \int_{0}^{b-a} \Re_{\mu}(\Psi ; t) \mu^{\prime}(t) d t \leqslant \int_{0}^{b-a} \Re_{\omega}(\Psi ; t) \mu^{\prime}(t) d t .
\end{aligned}
$$

Из предложения 3.8 о единственности экстремалей и $\omega$-перестановок задачи $(\star)$ вытекает, что мы имеем или два равенства в $(5.7)$ и $\Re_{\omega}(\Psi ; \cdot) \equiv \Re_{\mu}(\Psi ; \cdot)$, или строгие неравенства в (5.7). В последнем случае, положив $h_{1}=\omega^{\prime}$ и $h_{2}=\mu^{\prime}$ в 
(5.6), получаем утверждение (i). Отношение (ii) следует из сопоставления неравенства (ii) из следствия 5.2 и неравенства (5.5):

$$
\begin{gathered}
\int_{0}^{b-a} \Re_{\omega}(\Psi ; t) \omega^{\prime}(t) d t \leqslant \int_{0}^{b-a} \Sigma(\Psi ; t) \omega^{\prime}(t) d t, \\
\int_{0}^{b-a} \Re_{\omega}(\Psi ; t) d t \geqslant \int_{0}^{b-a} \Sigma(\Psi ; t) d t .
\end{gathered}
$$

Равенство $\int_{0}^{b-a} \Psi(t) d t=\int_{0}^{b-a} \Sigma(\Psi ; t) d t$ влечет за собой (iii). Наконец, из следствия 5.5 , замечания 5.6 и отношения $\Sigma(\Psi ; \cdot) \rightarrow \Re_{\omega}(\Psi ; \cdot)$ вытекает неравенство

$$
\int_{0}^{b-a} \Re_{\omega}(\Psi ; t) \omega^{\prime}(t) d t \leqslant \int_{0}^{b-a} \Sigma(\Psi ; t) \omega^{\prime}(t) d t .
$$

Ввиду неравенства (ii) из следствия 5.2 и предложения 3.8 равенство в (5.8) имеет место тогда и только тогда, когда $\Re_{\omega}(\Psi ; \cdot) \equiv \Sigma(\Psi ; \cdot)$.

Вспомним структуру производной $\Re_{\omega}^{\prime}(\Psi ; x) \omega$-перестановки, проясненную в разд. 4.3. Проверка следующей ее поточечной оценки через $\Sigma\left(\Psi^{\prime \prime} ; \cdot\right)$ проводится по схеме Корнейчука из доказательства соответствующего результата для $\Sigma$-перестановок в [6].

ПредЛОЖениЕ 5.12. Если $\Psi \in \mathbb{A C}^{2}[a, b] \cap \mathscr{M}_{l}^{0}[a, b], \Psi^{\prime \prime} \in \mathscr{M}_{n}^{0}[a, b] u n, l \in \mathbb{N}$, mo

$$
\left|\Re_{\omega}^{\prime}(\Psi ; x)\right| \leqslant \frac{1}{4} \int_{0}^{x} \Sigma\left(\Psi^{\prime \prime} ; t\right) d t, \quad x \in[0, b-a] .
$$

ДокАЗАТЕльство. Пусть $\left\{\left(t_{i}, \tau_{i}\right)\right\}_{i=1}^{m}:=\widetilde{\Lambda}(x)$, где множество $\widetilde{\Lambda}(x)$ введено в (4.22). В частности, $a \leqslant t_{1}<\tau_{1}=t_{1}+x<t_{2}<\tau_{2}=t_{2}+x<\cdots<t_{m}<\tau_{m}=$ $t_{m}+x \leqslant b$, и ввиду формулы (4.24) и следствия 2.3

Положим

$$
\Re_{\omega}^{\prime}(\Psi ; x) \leqslant \frac{1}{4} \sum_{i=1}^{m}\left|\Psi^{\prime}\left(t_{i}\right)-\Psi^{\prime}\left(\tau_{i}\right)\right|=\frac{1}{4} \sum_{i=1}^{m}\left|\int_{t_{i}}^{\tau_{i}} \Psi^{\prime \prime}(t) d t\right| .
$$

$$
I_{\nu}:=\int_{t_{\nu}}^{\tau_{\nu}} \Psi^{\prime \prime}(t) d t=\Psi^{\prime}\left(\tau_{\nu}\right)-\Psi^{\prime}\left(t_{\nu}\right), \quad \nu=1, \ldots, m .
$$

Поскольку $\operatorname{sign} \Psi^{\prime}\left(t_{\nu}\right)=-\operatorname{sign} \Psi^{\prime}\left(\tau_{\nu}\right)$,

$$
I_{\nu} \cdot \Psi^{\prime}\left(t_{\nu}\right)<0, \quad I_{\nu} \cdot \Psi^{\prime}\left(\tau_{\nu}\right)>0 .
$$

Для всех $\nu=1, \ldots, m$ выберем $t_{\nu}^{\prime}, \tau_{\nu}^{\prime}, t_{\nu}<t_{\nu}^{\prime} \leqslant \tau_{\nu}^{\prime} \leqslant \tau_{\nu}$, таким образом, что

$$
\operatorname{sign} I_{\nu} \cdot \int_{t_{\nu}^{\prime}}^{\tau_{\nu}^{\prime}} \Psi^{\prime \prime}(t) d t \geqslant\left|I_{\nu}\right|, \quad I_{\nu} \cdot \Psi^{\prime \prime}\left(t_{\nu}^{\prime}\right) \geqslant 0, \quad I_{\nu} \cdot \Psi^{\prime \prime}\left(\tau_{\nu}^{\prime}\right) \geqslant 0 .
$$

Покажем, что любые два интервала $\left(t_{\nu}^{\prime}, \tau_{\nu}^{\prime}\right)$ и $\left(t_{\nu+1}^{\prime}, \tau_{\nu+1}^{\prime}\right)$ при $\nu=1, \ldots, m-1$ разделены нулем производной $\Psi^{\prime \prime}$. Если $I_{\nu} \cdot I_{\nu+1}<0$, это следует из нашего выбора точек $t_{\nu}^{\prime}, \tau_{\nu}^{\prime}$ (см. (5.13)). Пусть $I_{\nu} \cdot I_{\nu+1}>0$. С одной стороны, согласно (5.13), $I_{\nu} \cdot \Psi^{\prime \prime}\left(\tau_{\nu}^{\prime}\right) \geqslant 0$ и $I_{\nu} \cdot \Psi^{\prime \prime}\left(t_{\nu}^{\prime}\right) \geqslant 0$. С другой стороны, ввиду $(5.12)$

$$
\operatorname{sign} I_{\nu} \cdot \int_{\tau_{\nu}^{\prime}}^{t_{\nu+1}^{\prime}} \Psi^{\prime \prime}(x) d x=\operatorname{sign} I_{\nu+1} \Psi^{\prime}\left(t_{\nu+1}^{\prime}\right)-\operatorname{sign} I_{\nu} \Psi^{\prime}\left(\tau_{\nu}^{\prime}\right)<0 ;
$$


следовательно, $\Psi^{\prime \prime}$ имеет нуль на $\left(\tau_{\nu}^{\prime}, t_{\nu+1}^{\prime}\right)$. Поскольку $\tau_{\nu}^{\prime}-t_{\nu}^{\prime} \leqslant \tau_{\nu}-t_{\nu} \leqslant x$ для всех $\nu=1, \ldots, m$, мы можем применить соотношения (5.10), (5.13) и лемму 4.5 в цепочке неравенств

$$
\left|\Re_{\omega}^{\prime}(\Psi ; x)\right| \leqslant \frac{1}{4} \sum_{\nu=1}^{m}\left|I_{\nu}\right| \leqslant \frac{1}{4} \sum_{\nu=1}^{m}\left|\int_{t_{\nu}^{\prime}}^{\tau_{\nu}^{\prime}} \Psi^{\prime \prime}(t) d t\right| \leqslant \frac{1}{4} \int_{0}^{x} \Sigma\left(\Psi^{\prime \prime} ; t\right) d t,
$$

которая завершает доказательство неравенства (5.9).

ЗАмЕчАнИЕ 5.13. Неравенство (5.9) точно в том смысле, что существуют функции $\Psi$, реализующие в нем знак равенства. Действительно, для $n \in \mathbb{N}$ пусть $f$ - любая $2(b-a) / n$-периодическая нечетная функция на $\mathbb{R}$ (например, $\sin (\pi n x /(b-a)))$ со свойствами

$$
f^{\prime \prime \prime}(t)<0, \quad f^{\prime \prime}\left(\frac{b-a}{2 n}+t\right)=f^{\prime \prime}\left(\frac{b-a}{2 n}-t\right), \quad t \in\left(0, \frac{b-a}{2 n}\right) .
$$

Пусть $K(t):=f(t-a), t \in[a, b]$. Тогда для $l=0,1$ и $x \in[0,(b-a) / n]$ мы имеем

$$
\Re_{\omega}\left(K^{(2 l)} ; x\right)=\Sigma\left(K^{(2 l)} ; x\right)=(-1)^{l} n K^{(2 l)}\left(\frac{x}{2}+\frac{b-a}{2 n}+a\right) .
$$

Согласно (5.15) и (5.16), следующие равенства имеют место для всех $x \in(0,(b-$ a) $/ n]$ :

$$
\begin{aligned}
\Re_{\omega}^{\prime}(K ; x) & =\frac{1}{4} n\left(K^{\prime}\left(\frac{b-a}{2 n}+\frac{x}{2}+a\right)-K^{\prime}\left(\frac{b-a}{2 n}-\frac{x}{2}+a\right)\right) \\
& =\frac{1}{2} n K^{\prime}\left(\frac{b-a}{2 n}+\frac{x}{2}+a\right)=\frac{1}{4} \int_{0}^{x} \Sigma\left(K^{\prime \prime} ; t\right) d t .
\end{aligned}
$$

В заключение, автор выражает глубокую благодарность профессорам Б. С. Митягину, В. М. Тихомирову и А. Г. Костюченко за внимание к работе.

\section{ЛИТЕРАТУРА}

1. Багдасаров C. K. Максимизация функционалов в $H^{\omega}[a, b]$. Матем. сб., 189, № 2, 3-72 (1998).

2. Багдасаров C. K. Экстремальные функции интегральных функционалов. Изв. PAH, № 2 (1999).

3. Bagdasarov $S$. K. Chebyshev Splines and Kolmogorov Inequalities. Operator Theory Advances and Applications, vol. 105, Birkhäuser Verlag, 1998.

4. Bennett $C$., Sharpley R. Interpolation of Operators. Academic Press, New York, 1988.

5. Hardy G. H., Littlewood J., Pölya G. Inequalities. Cambridge Univ. Press, New York, 1934.

6. Korneichuk N. P. Exact Constants in Approximation Theory, Encyclopedia of mathematics and its applications, Vol. 38, Cambridge Univ. Press, Cambridge, New York, 1990.

7. Крейн С. Г., Петунин Ю. И., Семенов Е. М. Интерполяция линейных операторов. Наука, М., 1978.

8. Nikol'skii S. M. La série de Fourier d'une fonction dont le module de continuité est donné, ДАН СССР, 52, 191-194, 1946. 